\title{
IFP-Intuitionistic Fuzzy Soft $h$-Ideals of Hemirings and Its Decision Making
}

\author{
Qi Liu and Jianming Zhan \\ Department of Mathematics, Hubei University for Nationalities, Enshi, Hubei 445000, China \\ Correspondence should be addressed to Jianming Zhan; zhanjianming@hotmail.com
}

Received 13 November 2013; Accepted 30 January 2014; Published 20 March 2014

Academic Editor: Tai-Ping Chang

Copyright ( 2014 Q. Liu and J. Zhan. This is an open access article distributed under the Creative Commons Attribution License, which permits unrestricted use, distribution, and reproduction in any medium, provided the original work is properly cited.

\begin{abstract}
The aim of this paper is to introduce the concepts of IFP-intuitionistic fuzzy soft $h$-ideals and IFP-equivalent intuitionistic fuzzy soft $h$-ideals of hemirings. Some characterizations and properties of them are given. In particular, some good examples are explored. Finally, we investigate aggregate intuitionistic fuzzy $h$-ideals of hemirings based on decision making.
\end{abstract}

\section{Introduction}

Dealing with problems in different areas of applied mathematics and information sciences, we have found that semirings are become more and more useful. They play an important role in studying optimization theory, graph theory, matrices, theory of discrete event dynamical systems, generalized fuzzy computation, and so on. The ideals of semirings play a central role in the structure theory, and the ideals of semirings do not in general coincide with the usual ring ideals; therefore, the usage of ideals in semirings is limited. In order to overcome the difficulty, many researchers mean a special semiring with a zero and with a commutative addition. We call this semiring hemiring. The properties of $h$ ideals in hemirings were thoroughly investigated by LaTorre [1] and by using the $h$-ideals, he established some analogues ring theorems for hemirings. In 2004, Jun et al. [2] introduced the concept of fuzzy $h$-ideals in hemirings and gave some related properties. In particular, Zhan and Dudek [3] studied the $h$-hemiregular hemirings. Some characterizations of $h$ semisimple and $h$-intra-hemirings were further investigated by Yin et al. $[4,5]$. In order to continue these papers, Ma et al. [6-8] investigated some generalized fuzzy $h$-ideals of hemirings. Zhan and Shum [9] has studied intuitionistic fuzzy $h$-ideals of hemirings. The other important results on semirings (hemirings) were discussed by Dudek et al. [10].

Mathematical modelling and manipulation of some kinds of uncertainties have become an increasingly important issue in solving complicated problems arising in a wide range of areas, such as, economies, engineering, medicine, environmental science, and information science. In 1999, Molodtsov [11] firstly introduced the soft set theory as a general mathematical tool for dealing with uncertainty and vagueness. Since then some researchers studied the operations of soft sets and their various applications [12-19]. Recently, some researchers investigated fuzzy soft sets with parameterizations, such as, fuzzy parameterized soft sets (FPsoft sets) [20], fuzzy parameterized fuzzy soft sets (FP-fuzzy soft sets) [20], intuitionistic fuzzy parameterized (IFP) soft set, intuitionistic fuzzy parameterized (IFP) fuzzy soft set, and intuitionistic fuzzy parameterized- (IFP-) intuitionistic fuzzy soft set [21].

In this paper, we introduce the concept of IFPintuitionistic soft $h$-ideals of hemirings and discuss some related results. Finally, we give some examples to show that the methods can be successfully applied to some uncertain problems.

\section{Preliminaries}

A semiring is an algebraic system $(S,+, \cdot)$ consisting of a nonempty set $S$ together with two binary operations on $S$ called addition and multiplication (denoted in the usual manner) such that $(S,+)$ and $(S, \cdot)$ are semigroups and the following distributive laws

$$
a(b+c)=a b+a c, \quad(a+b) c=a c+b c
$$


By zero of a semiring $(S,+, \cdot)$, we mean an element $0 \in S$ such that $0 \cdot x=x \cdot 0=0$ and $0+x=x+0=0$ for all $x \in S$. A semiring with zero and a commutative semigroup $(S,+)$ is called a hemiring. For the sake of simplicity, we will write $a b$ for $a \cdot b(a, b \in S)$.

A subhemiring of a hemiring $S$ is a subset $A$ of $S$ closed under addition and multiplication. A subset $A$ of $S$ is called a left (right) ideal of $S$ if $A$ is closed under addition and $S A \subseteq$ $A(A S \subseteq A)$. A subset $A$ is called an ideal if it is both a left ideal and a right ideal.

A subhemiring (left ideal, right ideal, and ideal) $A$ of $S$ is called an $h$-subhemiring (left $h$-ideal, right $h$-ideal, and $h$ ideal) of $S$, respectively, if for any $x, z \in S, a, b \in A$, and $x+$ $a+z=b+z$ implies $x \in A$.

From now on, $S$ is a hemiring, $U$ is an initial universe, $E$ is a set of parameters, $P(U)$ is the power set of $U$, and $A, B, C \subseteq$ E.

Definition 1 (see [22]). Let $U$ be an initial universe. A fuzzy set $X$ over $U$ is a set defined by a function $\mu_{X}$ representing a mapping

$$
\mu_{X}: U \longrightarrow[0,1]
$$

Here, $\mu_{X}$ is called membership function of $X$ and the value $\mu_{X}(u)$ is called the grade of membership of $\mu \in U$. The value represents the degree of $u$ belonging to fuzzy set $X$. Thus, a fuzzy set $X$ over $U$ can be represented as follows:

$$
X=\left\{\left(u, \mu_{X}(u)\right): u \in U, \mu_{X}(u) \in[0,1]\right\} .
$$

Note that the set of all the fuzzy sets over $U$ will be denoted by $F(U)$.

Definition 2 (see [23]). An intuitionistic fuzzy set (IFS) $X$ in $U$ is defined as an object of the following form:

$$
X=\left\{\left(x, \mu_{X}(u), v_{X}(u)\right): u \in U\right\},
$$

where the functions $\mu_{X}: U \rightarrow[0,1]$ and $\nu_{X}: U \rightarrow$ $[0,1]$ define the degree of membership and the degree of nonmembership of the element $u \in U$, respectively, and for every $u \in U$,

$$
0 \leq \mu_{X}(u)+v_{X}(u) \leq 1 .
$$

In addition for all $u \in U, U=\{(u, 1,0): u \in U\}$, $\emptyset=\{(u, 0,1): u \in U\}$ are intuitionistic fuzzy universal and intuitionistic fuzzy empty set, respectively.

Definition 3 (see [3]). (i) A fuzzy set $\mu$ of $S$ is said to be a fuzzy left (right) ideal of $S$ if the following conditions hold for all $x, y \in S: \mu(x+y) \geq \min \{\mu(x), \mu(y)\}$, and $\mu(x y) \geq$ $\mu(y)(\mu(x y) \geq \mu(x))$.

Note that if $\mu$ is a fuzzy left (right) ideal of $S$, then $\mu(0) \geq$ $\mu(x)$ for all $x \in S$.

(ii) A fuzzy left (right) $h$-ideal $\mu$ of $S$ is defined to be a fuzzy left (right) ideal $\mu$ of $S$ if for any $a, b, x, z \in S, x+a+z=$ $b+z$ implies $\mu(x) \geq \min \{\mu(a), \mu(b)\}$.

A fuzzy set $\mu$ is said to be a fuzzy $h$-ideal of $S$ if it is both a fuzzy left $h$-ideal of $S$ and a fuzzy right $h$-ideal of $S$.
Definition 4 (see [9]). (i) An intuitionistic fuzzy set $X$ of $S$ is said to be an intuitionistic fuzzy left (right) ideal of $S$ if the following conditions hold for all $x, y \in S: \mu(x+y) \geq$ $\min \{\mu(x), \mu(y)\}, \nu(x+y) \leq \max \{\nu(x), \nu(y)\}$ and $\mu(x y) \geq$ $\mu(y)(\mu(x y) \geq \mu(x)), \nu(x y) \leq \nu(y)(\nu(x y) \leq \nu(x))$.

Note that if $\mu$ is an intuitionistic fuzzy left (right) ideal of $S$, then $\mu(0) \geq \mu(x)$ and $\nu(0) \leq \nu(x)$ for all $x \in S$.

(ii) An intuitionistic fuzzy left (right) $h$-ideal $X$ of $S$ is defined to be an intuitionistic fuzzy left (right) ideal $X$ of $S$ if for any $a, b, x, z \in S, x+a+z=b+z$ implies $\mu(x) \geq$ $\min \{\mu(a), \mu(b)\}$ and $\nu(x) \leq \max \{\nu(a), \nu(b)\}$.

An intuitionistic fuzzy set $X$ is said to be an intuitionistic fuzzy $h$-ideal of $S$ if it is both an intuitionistic fuzzy left $h$-ideal and an intuitionistic fuzzy right $h$-ideal of $S$.

The following concepts are cited in [21].

Definition 5. Let $U$ be an initial universe, $E$ the set of all parameters, and $X$ an intuitionistic fuzzy set over $E$ with the membership function $\mu_{X}: E \rightarrow[0,1]$ and nonmembership function $\nu_{X}: E \rightarrow[0,1]$ and $\omega_{X}$ is an intuitionistic fuzzy set over $U$ for all $x \in E$. Then, an $\Omega$-set $\Omega_{X}$ over $\operatorname{IF}(U)$ is a set defined by a function $\omega_{X}(x)$ representing a mapping

$$
\omega_{X}: E \longrightarrow \operatorname{IF}(U) \text { such that } \omega_{X}(x)=\emptyset \quad \text { if } x \notin X \text {. }
$$

Here, $\omega_{X}$ is called intuitionistic fuzzy approximation of $\Omega$-set $\Omega_{X}, \omega_{X}(x)$ is an intuitionistic fuzzy set called $x$-element of the $\Omega$-set for all $x \in E$. Thus, an $\Omega$-set $\Omega_{X}$ over $U$ can be represented by the set of ordered pairs

$$
\Omega_{X}=\left\{\left(\frac{\left(\mu_{X}(x), v_{X}(x)\right)}{x}, \omega_{X}(x)\right): x \in E, \omega_{X}(x) \in \operatorname{IF}(U)\right\} .
$$

Note that, if $\mu_{X}(x)=0, \nu_{X}(x)=1$ and $\omega_{X}(x)=\emptyset$, we do not display such elements in the set. Also, it must be noted that the sets of all $\Omega$-sets over $\operatorname{IF}(U)$ will be denoted by $\Omega(U)$.

Definition 6. Let $\Omega_{X} \in \Omega(U)$.

(i) If $\Omega_{X}(x)=\emptyset$ for all $x \in E$, then $\Omega_{X}$ is called an $X$ empty $\Omega$-set, denoted by $\Omega_{\emptyset_{X}}$.

(ii) If $X=\emptyset$, then the $X$-empty $\Omega$-set $\left(\Omega_{\emptyset_{X}}\right)$ is called empty $\Omega$-set, denoted by $\Omega_{\emptyset}$. Here, $\emptyset$ means intuitionistic fuzzy empty set.

(iii) If $\mu_{X}(x)=1, v_{X}(x)=0$, and $\omega_{X}(x)=U$ for all $x \in X$, then $\Omega_{X}$ is called $X$-universal $\Omega$-set, denoted by $\Omega_{\widetilde{X}}$.

(iv) If $X$ is equal to intuitionistic fuzzy universal set over $E$, then the $X$-universal $\Omega$-set is called universal $\Omega$ set, denoted by $\Omega_{\widetilde{E}}$. Here, $U$ means that intuitionistic fuzzy universal set.

Definition 7. Let $\Omega_{X}, \Omega_{Y} \in \Omega(U)$. Then

(i) $\Omega_{X}$ is an IFP-intuitionistic fuzzy subset of $\Omega_{Y}$,

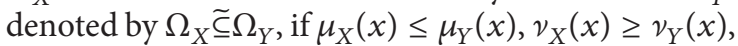
and $\omega_{X}(x) \subseteq \omega_{Y}(x)$ for all $x \in E$; 
(ii) $\Omega_{X}$ and $\Omega_{Y}$ are IFP-equal, denoted by $\Omega_{X}=\Omega_{Y}$, if $\mu_{X}(x)=\mu_{Y}(x), \nu_{X}(x)=\nu_{Y}(x)$, and $\gamma_{X}(x)=\gamma_{Y}(x)$ for all $x \in E$.

Definition 8. Let $\Omega_{X} \in \Omega(U)$. Then the complement of $\Omega_{X}$, denoted by $\Omega_{X}^{\bar{c}}$, is an IFP-intuitionistic fuzzy soft set defined by

$$
\begin{gathered}
\mu_{X}^{\bar{c}}(x)=1-\mu(x), \quad \nu_{X}^{\bar{c}}(x)=1-\nu(x), \\
\gamma_{X}^{\bar{c}}(x)=U \backslash \gamma_{X}(x) .
\end{gathered}
$$

Definition 9. Let $\Omega_{X}, \Omega_{Y} \in \Omega(U)$.

(i) The intersection of $\Omega_{X}$ and $\Omega_{Y}$, denoted by $\Omega_{X} \widetilde{\cap} \Omega_{Y}$, is defined by

$$
\Omega_{X} \widetilde{\cap} \Omega_{Y}=\left\{\left(\frac{\left(\mu_{X \tilde{\cap} Y}(x), \nu_{X \widetilde{\cup} Y}(x)\right)}{x}, \omega_{X \widetilde{\cap} Y}(x)\right) \mid x \in E\right\} .
$$

Here,

$$
\begin{aligned}
\mu_{X \tilde{ก} Y}(x)= & \min \left\{\mu_{X}(x), \mu_{Y}(x)\right\}, \\
\nu_{X \widetilde{\cup} Y}(x)= & \max \left\{v_{X}(x), v_{Y}(x)\right\}, \\
\omega_{X \widetilde{\cap} Y}(x)= & \left\{\left(u, \min \left\{\mu_{\omega_{X}(x)}(u), \mu_{\omega_{Y}(x)}(u)\right\},\right.\right. \\
& \left.\left.\max \left\{v_{\omega_{X}(x)}(u), v_{\omega_{Y}(x)}(u)\right\}\right) \mid u \in U\right\},
\end{aligned}
$$

where $\omega_{X \tilde{\cap} Y}(x)=\omega_{X}(x) \cap \omega_{Y}(x)$ for all $x \in E$.

(ii) The union of $\Omega_{X}$ and $\Omega_{Y}$, denoted by $\Omega_{X} \widetilde{U} \Omega_{Y}$, is defined by

$$
\Omega_{X} \widetilde{U} \Omega_{Y}=\left\{\left(\frac{\left(\mu_{X \widetilde{U} Y}(x), v_{X \widetilde{n} Y}(x)\right)}{x}, \omega_{X \widetilde{\cup}}(x)\right) \mid x \in E\right\} .
$$

Here,

$$
\begin{aligned}
\mu_{X \widetilde{\cup} Y}(x)= & \max \left\{\mu_{X}(x), \mu_{Y}(x)\right\}, \\
v_{X \tilde{n} Y}(x)= & \min \left\{v_{X}(x), v_{Y}(x)\right\}, \\
\omega_{X \widetilde{\cup} Y}(x)= & \left\{\left(u, \max \left\{\mu_{\omega_{X}(x)}(u), \mu_{\omega_{Y}(x)}(u)\right\},\right.\right. \\
& \left.\left.\min \left\{v_{\omega_{X}(x)}(u), v_{\omega_{Y}(x)}(u)\right\}\right) \mid u \in U\right\},
\end{aligned}
$$

where $\omega_{X \widetilde{Y} Y}(x)=\omega_{X}(x) \cup \omega_{Y}(x)$ for all $x \in E$.

(iii) The $\wedge$-product of $\Omega_{X}$ and $\Omega_{Y}$, denoted by $\Omega_{X} \wedge \Omega_{Y}$, is defined by

$$
\begin{aligned}
\Omega_{X} & \wedge \Omega_{Y} \\
& =\left\{\left(\frac{\left(\mu_{X \wedge Y}(x, y), v_{X \wedge Y}(x, y)\right)}{x}, \omega_{X \wedge Y}(x, y)\right) \mid x \in E\right\} .
\end{aligned}
$$

Here

$$
\begin{aligned}
\mu_{X \wedge Y}(x, y)= & \min \left\{\mu_{X}(x), \mu_{Y}(y)\right\}, \\
\nu_{X \vee Y}(x, y)= & \max \left\{\nu_{X}(x), \nu_{Y}(y)\right\}, \\
\omega_{X \wedge Y}(x)= & \left\{\left(u, \min \left\{\mu_{\omega_{X}(x)}(u), \mu_{\omega_{Y}(y)}(u)\right\},\right.\right. \\
& \left.\left.\max \left\{v_{\omega_{X}(x)}(u), v_{\omega_{Y}(y)}(u)\right\}\right) \mid u \in U\right\},
\end{aligned}
$$

where $\omega_{X \wedge Y}(x)=\omega_{X}(x) \wedge \omega_{Y}(x)$ for all $x \in E$.

\section{IFP-Intuitionistic Fuzzy Soft $h$-Ideals}

Definition 10. Let $S$ be a hemiring, $E$ a set of parameters, and $X$ an intuitionistic fuzzy set over $E, \Omega_{X}=$ $\left\{\left(\left(\mu_{X}(x), \nu_{X}(x)\right) / x, \omega_{X}(x)\right) \mid x \in E, \omega_{X}(x) \in \operatorname{IF}(U)\right\} \in \Omega(U)$.

Then $\Omega_{X}$ is said to be an intuitionistic fuzzy parameterized intuitionistic fuzzy soft $h$-ideal (briefly, IFP-intuitionistic fuzzy soft $h$-ideal) over $S$, if for any $x \in E, \omega_{X}(x)$ is an intuitionistic fuzzy $h$-ideal of $S$.

Example 11. Let $S=\mathbb{Z}_{3}=\{0,1,2\}$ be a hemiring and $E=$ $\{a, b\}$ be a set of parameters.

If $X=\{(0.3,0.6) / a,(0.5,0.2) / b\}, \omega_{X}(a)=\{(0.7,0.2) / 0$, $(0.6,0.3) / 1,(0.6,0.3) / 2\}, \omega_{X}(b)=\{(0.5,0.1) / 0,(0.3,0.6) / 1$, $(0.3,0.6) / 2\}$, then $\Omega_{X}$ is an IFP-intuitionistic fuzzy soft $h$ ideal over $S$.

Example 12. Let $S=M_{n}$, where $M_{n}$ is a matrix hemiring, $E=\{a, b\}$, the meaning of the parameters $a, b$, respectively. If $X$ is an intuitionistic fuzzy set over $S$ defined by

$$
\begin{aligned}
& \mu_{X}(x)= \begin{cases}0.2, & x=a, \\
0.6, & x=b,\end{cases} \\
& \nu_{X}(x)=1-\mu_{X}(x) .
\end{aligned}
$$

Define $\omega_{X}$ by

$$
\begin{aligned}
& \mu_{\omega_{X}(a)}(r)= \begin{cases}0, & r \text { is not scalar matrix, } \\
1, & r \text { is scalar matrix, }\end{cases} \\
& v_{\omega_{X}(a)}(r)=1-\mu_{\omega_{X}(a)}(r) . \\
& \mu_{\omega_{X}(b)}(r)= \begin{cases}0, & r \text { is not upper triangular matrix, } \\
1, & r \text { is upper triangular matrix, }\end{cases} \\
& v_{\omega_{X}(b)}(r)=1-\mu_{\omega_{X}(b)}(r) .
\end{aligned}
$$

For all $x \in E$, we can verify that $\omega_{X}(x)$ is an intuitionistic fuzzy left $h$-ideal of $S$ and, hence, $\Omega_{X}$ is an IFP-intuitionistic fuzzy soft left $h$-ideal over $S$.

Proposition 13. If $\Omega_{X}$ and $\Omega_{Y}$ are IFP-intuitionistic fuzzy soft $h$-ideals over $S$, then their intersection $\Omega_{X} \widetilde{\bigcap} \Omega_{Y}$ is still an IFPintuitionistic fuzzy soft $h$-ideal over $S$.

Proof. We can write $\Omega_{X} \cap \Omega_{Y}=\Omega_{X \tilde{n} Y}$. For all $x \in E$, $\mu_{X \tilde{ก} Y}(x)=\min \left\{\mu_{X}(x), \mu_{Y}(x)\right\}, \nu_{X \widetilde{\cup} Y}(x)=\max \left\{\mu_{X}(x)\right.$, 
$\left.\mu_{Y}(x)\right\}$. Now we will prove $\omega_{X}(x) \cap \omega_{Y}(x)$ is a fuzzy $h$-ideal of $S$. For all $x \in E, s_{1}, s_{2} \in S$,

$$
\begin{aligned}
& \left(\mu_{\omega_{X}(x)} \cap \mu_{\omega_{Y}(x)}\right)\left(s_{1}+s_{2}\right) \\
& =\min \left\{\mu_{\omega_{X}(x)}\left(s_{1}+s_{2}\right), \mu_{\omega_{Y}(x)}\left(s_{1}+s_{2}\right)\right\} \\
& \geq \min \left\{\min \left\{\mu_{\omega_{X}(x)}\left(s_{1}\right), \mu_{\omega_{X}(x)}\left(s_{2}\right)\right\},\right. \\
& \left.\min \left\{\mu_{\omega_{Y}(x)}\left(s_{1}\right), \mu_{\omega_{Y}(x)}\left(s_{2}\right)\right\}\right\} \\
& =\min \left\{\min \left\{\mu_{\omega_{X}(x)}\left(s_{1}\right), \mu_{\omega_{Y}(x)}\left(s_{1}\right)\right\},\right. \\
& \left.\min \left\{\mu_{\omega_{X}(x)}\left(s_{2}\right), \mu_{\omega_{Y}(x)}\left(s_{2}\right)\right\}\right\} \\
& =\min \left\{\left(\mu_{\omega_{X}(x)} \cap \mu_{\omega_{Y}(x)}\right)\left(s_{1}\right),\left(\mu_{\omega_{X}(x)} \cap \mu_{\omega_{Y}(x)}\right)\left(s_{2}\right)\right\} \text {; } \\
& \left(v_{\omega_{X}(x)} \cup v_{\omega_{Y}(x)}\right)\left(s_{1}+s_{2}\right) \\
& =\max \left\{v_{\omega_{X}(x)}\left(s_{1}+s_{2}\right), v_{\omega_{Y}(x)}\left(s_{1}+s_{2}\right)\right\} \\
& \leq \max \left\{\max \left\{v_{\omega_{X}(x)}\left(s_{1}\right), v_{\omega_{X}(x)}\left(s_{2}\right)\right\},\right. \\
& \left.\max \left\{v_{\omega_{Y}(x)}\left(s_{1}\right), v_{\omega_{Y}(x)}\left(s_{2}\right)\right\}\right\} \\
& =\max \left\{\max \left\{v_{\omega_{X}(x)}\left(s_{1}\right), v_{\omega_{Y}(x)}\left(s_{1}\right)\right\},\right. \\
& \left.\max \left\{v_{\omega_{X}(x)}\left(s_{2}\right), v_{\omega_{Y}(x)}\left(s_{2}\right)\right\}\right\} \\
& =\max \left\{\left(v_{\omega_{X}(x)} \cup v_{\omega_{Y}(x)}\right)\left(s_{1}\right),\left(v_{\omega_{X}(x)} \cup v_{\omega_{Y}(x)}\right)\left(s_{2}\right)\right\} .
\end{aligned}
$$

Since $\mu_{\omega_{X}(x)}\left(s_{1} s_{2}\right) \geq \mu_{\omega_{X}(x)}\left(s_{2}\right), \nu_{\omega_{X}(x)}\left(s_{1} s_{2}\right) \leq \nu_{\omega_{X}(x)}\left(s_{2}\right)$ and $\mu_{\omega_{Y}(x)}\left(s_{1} s_{2}\right) \geq \mu_{\omega_{Y}(x)}\left(s_{2}\right), v_{\omega_{Y}(x)}\left(s_{1} s_{2}\right) \leq v_{\omega_{Y}(x)}\left(s_{2}\right)$, then

$$
\begin{aligned}
\left(\mu_{\omega_{X}(x)} \cap \mu_{\omega_{Y}(x)}\right)\left(s_{1} s_{2}\right) & =\min \left\{\mu_{\omega_{X}(x)}\left(s_{1} s_{2}\right), \mu_{\omega_{Y}(x)}\left(s_{1} s_{2}\right)\right\} \\
& \geq \min \left\{\mu_{\omega_{X}(x)}\left(s_{2}\right), \mu_{\omega_{Y}(x)}\left(s_{2}\right)\right\} \\
& =\left(\mu_{\omega_{X}(x)} \cap \mu_{\omega_{Y}(x)}\right)\left(s_{2}\right)
\end{aligned}
$$$$
\left(v_{\omega_{X}(x)} \cup v_{\omega_{Y}(x)}\right)\left(s_{1} s_{2}\right)=\max \left\{v_{\omega_{X}(x)}\left(s_{1} s_{2}\right), v_{\omega_{Y}(x)}\left(s_{1} s_{2}\right)\right\}
$$$$
\leq \max \left\{v_{\omega_{X}(x)}\left(s_{2}\right), v_{\omega_{Y}(x)}\left(s_{2}\right)\right\}
$$$$
=\left(v_{\omega_{X}(x)} \cup v_{\omega_{Y}(x)}\right)\left(s_{2}\right) \text {. }
$$

Thus we see $\omega_{X}(x) \cap \omega_{Y}(x)$ is an intuitionistic fuzzy left ideal of $S$.

Likewise, the proof of right ideal of $S$ can be made in a similar way.
For all $x \in E, a, b, s, z \in S$, let $s+a+z=b+z$; then

$$
\begin{aligned}
& \left(\mu_{\omega_{X}(x)} \cap \mu_{\omega_{Y}(x)}\right)(s) \\
& =\min \left\{\mu_{\omega_{X}(x)}(s), \mu_{\omega_{Y}(x)}(s)\right\} \\
& \geq \min \left\{\min \left\{\mu_{\omega_{X}(x)}(a), \mu_{\omega_{X}(x)}(b)\right\},\right. \\
& \left.\min \left\{\mu_{\omega_{Y}(x)}(a), \mu_{\omega_{Y}(x)}(b)\right\}\right\} \\
& =\min \left\{\min \left\{\mu_{\omega_{X}(x)}(a), \mu_{\omega_{Y}(x)}(a)\right\},\right. \\
& \left.\quad \min \left\{\mu_{\omega_{X}(x)}(b), \mu_{\omega_{Y}(x)}(b)\right\}\right\} \\
& =\min \left\{\left(\mu_{\omega_{X}(x)} \cap \mu_{\omega_{Y}(x)}\right)(a),\left(\mu_{\omega_{X}(z)} \cap \mu_{\omega_{Y}(z)}\right)(b)\right\} ; \\
& \left(v_{\omega_{X}(x)} \cup v_{\omega_{Y}(x)}\right)(s) \\
& =\max \left\{v_{\omega_{X}(x)}(s), v_{\omega_{Y}(x)}(s)\right\} \\
& \leq \max \left\{\max \left\{v_{\omega_{X}(x)}(a), v_{\omega_{X}(x)}(b)\right\},\right. \\
& \left.\max \left\{v_{\omega_{Y}(x)}(a), v_{\omega_{Y}(x)}(b)\right\}\right\} \\
& =\max \left\{\left(v_{\omega_{X}(x)} \cup v_{\omega_{Y}(x)}\right)(a),\left(v_{\omega_{X}(x)} \cup v_{\omega_{Y}(x)}\right)(b)\right\} . \\
& \left.=\max \left\{\max \left\{v_{\omega_{X}(x)}(a), v_{\omega_{Y}(x)}(a)\right\}, v_{\omega_{Y}(x)}(b)\right\}\right\}
\end{aligned}
$$

Hence $\omega_{X}(x) \cap \omega_{Y}(x)$ is an intuitionistic fuzzy $h$-ideal of $S$; that is to say, $\omega_{X \tilde{\cap} Y}(x)=\omega_{X}(x) \cap \omega_{Y}(x)$ is an intuitionistic fuzzy $h$-ideal of $S$. Therefore, $\Omega_{X} \widetilde{\bigcap} \Omega_{Y}$ is an IFP-intuitionistic fuzzy soft $h$-ideal over $S$.

We know that the intersection of all IFP-intuitionistic fuzzy soft $h$-ideals over $S$ is also an IFP-intuitionistic fuzzy soft $h$-ideal over $S$. Then we would consider whether the union of IFP-intuitionistic fuzzy soft $h$-ideals over $S$ is also an IFP-intuitionistic fuzzy soft $h$-ideal over $S$.

Notation 1. Let $\Omega_{X}$ and $\Omega_{Y}$ be IFP-intuitionistic fuzzy soft $h$ ideals over $S$. Then we said the sequence of values is ordered, if for any $x \in E, s_{1}, s_{2} \in S$ : (i) $\mu_{\omega_{X}(x)}\left(s_{1}\right) \geq \mu_{\omega_{X}(x)}\left(s_{2}\right)$ implies $\mu_{\omega_{Y}(x)}\left(s_{1}\right) \geq \mu_{\omega_{Y}(x)}\left(s_{2}\right)$, (ii) $v_{\omega_{X}(x)}\left(s_{1}\right) \geq v_{\omega_{X}(x)}\left(s_{2}\right)$ implies $v_{\omega_{Y}(x)}\left(s_{1}\right) \geq v_{\omega_{Y}(x)}\left(s_{2}\right)$

Proposition 14. Let $\Omega_{X}$ and $\Omega_{Y}$ be IFP-intuitionistic fuzzy soft $h$-ideals over $S$ with ordered sequence of values. Then their union $\Omega_{X} \widetilde{\bigcup} \Omega_{Y}$ is still an IFP-intuitionistic fuzzy soft $h$-ideal over $S$.

Proof. The proof is similar to the proof of Proposition 13.

Proposition 15. Let $\Omega_{X}$ and $\Omega_{Y}$ be IFP-intuitionistic fuzzy soft h-ideals over $S$. Then $\Omega_{X} \wedge \Omega_{Y}$ is an IFP-intuitionistic fuzzy soft $h$-ideal over $S \times S$.

Proof. The proof is similar to the proof of Proposition 13. 
Definition 16. Let $\Omega_{X}$ be an IFP-intuitionistic fuzzy soft $h$ ideal over $S$ and $\Omega_{Y}$ an IFP-intuitionistic fuzzy soft subset over $S$. Then $\Omega_{X}$ is said to be an IFP-intuitionistic fuzzy soft $h$-ideal of $\Omega_{Y}$, if $\omega_{X}$ is an IFP-intuitionistic fuzzy soft subset of $\omega_{Y}$.

Example 17. Let $S=\mathbb{Z}_{4}=\{0,1,2,3\}$ be a hemiring and $E=$ $\{a, b\}$ a set of parameters. If $X=\{(0.1,0.7) / a,(0.3,0.6) / b\}$, $\omega_{X}(a)=\{(0.4,0.5) / 0,(0.2,0.7) / 1,(0.3,0.6) / 2,(0.2,0.7) / 3\}$, and $\omega_{X}(b)=\{(0.6,0.2) / 0,(0.1,0.6) / 1,(0.3,0.5) / 2,(0.1,0.6)$ $/ 3\}$ and $Y=\{(0.5,0.3) / a,(0.6,0.3) / b\}, \omega_{Y}(a)=\{(0.5,0.2) /$ $0,(0.3,0.6) / 1,(0.4,0.4) / 2,(0.2,0.6) / 3\}$, and $\omega_{Y}(b)=\{(0.9,0) /$ $0,(0.8,0.2) / 1,(0.9,0.1) / 2,(0.7,0.3) / 3\}$, then $\Omega_{X}$ is an IFPintuitionistic fuzzy soft $h$-ideal over $S, \Omega_{Y}$ is an IFPintuitionistic fuzzy soft subset over $S$, and $\Omega_{X}$ is an IFPintuitionistic fuzzy soft $h$-ideal of $\Omega_{Y}$.

Theorem 18. Let $\Omega_{X}, \Omega_{Y}$, and $\Omega_{Z}$ be IFP-intuitionistic fuzzy soft set over S. If $\Omega_{X}$ is an IFP-intuitionistic fuzzy soft h-ideal of $\Omega_{Y}$ and $\Omega_{Y}$ is an IFP-intuitionistic fuzzy soft h-ideal of $\Omega_{Z}$, then $\Omega_{X}$ is an IFP-intuitionistic fuzzy soft $h$-ideal of $\Omega_{Z}$.

Proof. The proof is obvious; therefore omit it.

\section{IFP-Equivalent Intuitionistic Fuzzy Soft $h$-Ideals}

Definition 19. Let $\Omega_{X}=\left\{\left(\left(\mu_{X}(x), \nu_{X}(x)\right) / x, \omega_{X}(x)\right): x \in\right.$ $\left.A, \omega_{X}(x) \in \operatorname{IF}(S)\right\}$ and let $\Omega_{Y}=\left\{\left(\left(\mu_{Y}(y), \nu_{Y}(y)\right) / y, \omega_{Y}(y)\right):\right.$ $\left.y \in B, \omega_{Y}(y) \in \operatorname{IF}(K)\right\}$ be an IFP-intuitionistic fuzzy soft $h$ ideal over hemirings $S$ and $K$, respectively. If $f: S \rightarrow K$ and $g: A \rightarrow B$ are two functions, then $(f, g)$ is called an IFPintuitionistic fuzzy soft homomorphism such that $(f, g)$ is an IFP-intuitionistic fuzzy soft homomorphism over $h$-ideal from $\Omega_{X}$ to $\Omega_{Y}$. The latter is written by $\Omega_{X} \sim \Omega_{Y}$ if the following conditions are satisfied:

(1) $f$ is an epimorphism from $S$ to $K$,

(2) $g$ is a surjective mapping,

(3) $\mu_{X}(x)=\mu_{Y}(g(x)), v_{X}(x)=\nu_{Y}(g(x))$, and $f\left(\omega_{X}(x)\right)=$ $\omega_{Y}(g(x))$ for all $x \in A$.

In the above definition, if $f$ is an isomorphism from $S$ to $K$ and $g$ is a bijective mapping, then $(f, g)$ is called an IFPintuitionistic fuzzy soft isomorphism so that $(f, g)$ is an IFPintuitionistic fuzzy soft isomorphism over $h$-ideal from $\Omega_{X}$ to $\Omega_{Y}$, denoted by $\Omega_{X} \simeq \Omega_{Y}$.

Example 20. Let $S=(\mathbb{Z},+, \times)$ and $K=(2 \mathbb{Z},+, \times), A=\{1,2\}$, and $B=\{3,6\}$. Define a homomorphism $f$ from $S$ onto $K$ by $f(s)=2 s$ for $r \in S$, and a mapping $g$ from $A$ onto $B$ by $g(x)=3 x$, for $x \in A$.

Let $X$ be an intuitionistic fuzzy set over $A$ defined by $X=$ $\{(0.3,0.6) / 1,(0.7,0.2) / 2\}$ and $Y$ a fuzzy set over $B$ defined by $Y=\{(0.3,0.6) / 3,(0.7,0.2) / 6\}$.
Let $\omega_{X}: A \rightarrow F(S)$ defined by

$$
\begin{aligned}
& \left(\omega_{X}(1)\right)(s)= \begin{cases}(0.2,0.7) & s=2 k+1, k \in \mathbb{Z} ; \\
(0.6,0.3) & s=2 k, k \in \mathbb{Z} .\end{cases} \\
& \left(\omega_{X}(2)\right)(s)= \begin{cases}(0.3,0.6) & s=2 k+1, k \in \mathbb{Z} ; \\
(0.4,0.5) & s=2 k, k \in \mathbb{Z} .\end{cases}
\end{aligned}
$$

$\omega_{Y}: B \rightarrow F(K)$ defined by

$$
\begin{aligned}
& \left(\omega_{Y}(4)\right)(s)= \begin{cases}(0.2,0.7) & s=4 k+2, k \in \mathbb{Z} ; \\
(0.6,0.3) & s=4 k, k \in \mathbb{Z} .\end{cases} \\
& \left(\omega_{Y}(8)\right)(s)= \begin{cases}(0.3,0.6) & s=4 k+2, k \in \mathbb{Z} ; \\
(0.4,0.5) & s=4 k, k \in \mathbb{Z} .\end{cases}
\end{aligned}
$$

It is clear that $\Omega_{X}$ and $\Omega_{Y}$ are IFP-intuitionistic fuzzy soft $h$-ideals over $S$ and $K$, respectively. We can immediately see that $\mu_{X}(x)=\mu_{Y}(g(x))$ and we can deduce that $f\left(\omega_{X}(x)\right)=$ $\omega_{Y}(g(x))$ for all $x \in A$. Hence $(f, g)$ is an IFP-intuitionistic fuzzy soft homomorphism from $\Omega_{X}$ to $\Omega_{Y}$.

Lemma 21 (see [3]). If $f: S \rightarrow K$ is an epimorphism of hemirings and $X$ is an intuitionistic fuzzy $h$-ideal of $S$, then $f(X)$ is an intuitionistic fuzzy $h$-ideal of $K$.

Theorem 22. Let $\Omega_{X}=\left\{\left(\left(\mu_{X}(x), v_{X}(x)\right) / x, \omega_{X}(x)\right): x \in\right.$ $\left.A, \omega_{X}(x) \in I F(S)\right\}$ be an IFP-intuitionistic fuzzy soft $h$-ideal over $S$ and $\Omega_{Y}=\left\{\left(\left(\mu_{Y}(y), \nu_{Y}(y)\right) / y, \omega_{Y}(y)\right): y \in B, \omega_{Y}(y) \in\right.$ $\operatorname{IF}(K)\}$ an IFP-intuitionistic fuzzy soft set over hemiring $K$. If $\Omega_{X}$ is an IFP-intuitionistic fuzzy soft homomorphic to $\Omega_{Y}$, then $\Omega_{Y}$ is an IFP-intuitionistic fuzzy soft $h$-ideal over $K$.

Proof. Let $(f, g)$ be an IFP-intuitionistic fuzzy soft homomorphism from $\Omega_{X}$ to $\Omega_{Y}$. Since $\Omega_{X}$ is an IFP-intuitionistic fuzzy soft $h$-ideal over $S, f(S)=K$ and $\omega_{X}(x)$ is a fuzzy $h$-ideal of $S$ for all $x \in A$. Now, for all $y \in B$, there exists $x \in A$ such that $g(x)=y$, so $\mu_{Y}(y)=\mu_{Y}(g(x))=\mu_{X}(x), \nu_{Y}(y)=\nu_{Y}(g(x))=$ $\nu_{X}(x)$. By Lemma 21, $\omega_{Y}(y)=\omega_{Y}(g(x))=f\left(\omega_{X}(x)\right)$ is an intuitionistic fuzzy $h$-ideal of the hemiring $K$, so $\Omega_{Y}$ must be an IFP-intuitionistic fuzzy soft $h$-ideal over $K$ as well.

Definition 23. Let $\Omega_{X}=\left\{\left(\left(\mu_{X}(x), \nu_{X}(x)\right) / x, \omega_{X}(x)\right): x \in\right.$ $\left.E, \omega_{X}(x) \in \operatorname{IF}(S)\right\}$ be an IFP-intuitionistic fuzzy soft $h$-ideal over hemiring $S$. Then $\Omega_{X}$ is said to be an IFP-equivalent intuitionistic fuzzy soft $h$-ideal over $S$ if, for any $x, y \in E$, $\mu_{X}(x)=\mu_{X}(y)$, and $\nu_{X}(x)=\nu_{X}(y)$, we have $\omega_{X}(x)=\omega_{X}(y)$.

By the Definitions 19 and 5, we can easily obtain that $\Omega_{X}^{\bar{c}}$ is an IFP-equivalent intuitionistic fuzzy soft $h$-ideal over $S$, if $\Omega_{X}$ is an IFP-equivalent intuitionistic fuzzy soft $h$-ideal over $S$.

Example 24. Assume that

$$
S_{x y}=\left\{\left(\begin{array}{ll}
x & y \\
x & y
\end{array}\right) \mid x, y \in \mathbb{Z}_{2}=\{0,1\}\right\}
$$


is a hemiring, $E=\left\{x_{1}, x_{2}, x_{3}, x_{4}\right\}$ is a set of parameters, and $X$ is an intuitionistic fuzzy set over $S$ defined by $X=$ $\left\{(0.3,0.7) / x_{1},(0.6,0.2) / x_{2},(0.6,0.2) / x_{3},(0.7,0.1) / x_{4}\right\}$,

$$
\begin{aligned}
& \omega_{X}\left(x_{1}\right)=\left\{\frac{(0.7,0.1)}{\left(\begin{array}{ll}
0 & 0 \\
0 & 0
\end{array}\right)}, \frac{(0.3,0.6)}{\left(\begin{array}{ll}
0 & 1 \\
0 & 1
\end{array}\right)}, \frac{(0.3,0.6)}{\left(\begin{array}{ll}
1 & 0 \\
1 & 0
\end{array}\right)}, \frac{(0.6,0.2)}{\left(\begin{array}{ll}
1 & 1 \\
1 & 1
\end{array}\right)}\right\}, \\
& \omega_{X}\left(x_{1}\right)=\left\{\frac{(0.5,0.1)}{\left(\begin{array}{ll}
0 & 0 \\
0 & 0
\end{array}\right)}, \frac{(0.2,0.8)}{\left(\begin{array}{ll}
0 & 1 \\
0 & 1
\end{array}\right)}, \frac{(0.2,0.8)}{\left(\begin{array}{ll}
1 & 0 \\
1 & 0
\end{array}\right)}, \frac{(0.3,0.6)}{\left(\begin{array}{ll}
1 & 1 \\
1 & 1
\end{array}\right)}\right\}, \\
& \omega_{X}\left(x_{1}\right)=\left\{\frac{(0.5,0.1)}{\left(\begin{array}{ll}
0 & 0 \\
0 & 0
\end{array}\right)}, \frac{(0.2,0.8)}{\left(\begin{array}{ll}
0 & 1 \\
0 & 1
\end{array}\right)}, \frac{(0.2,0.8)}{\left(\begin{array}{ll}
1 & 0 \\
1 & 0
\end{array}\right)}, \frac{(0.3,0.6}{\left(\begin{array}{ll}
1 & 1 \\
1 & 1
\end{array}\right)}\right\}, \\
& \omega_{X}\left(x_{1}\right)=\left\{\frac{(0.8,0.1)}{\left(\begin{array}{ll}
0 & 0 \\
0 & 0
\end{array}\right)}, \frac{(0.5,0.4)}{\left(\begin{array}{ll}
0 & 1 \\
0 & 1
\end{array}\right)}, \frac{(0.5,0.4)}{\left(\begin{array}{ll}
1 & 0 \\
1 & 0
\end{array}\right)}, \frac{(0.7,0.3)}{\left(\begin{array}{ll}
1 & 1 \\
1 & 1
\end{array}\right)}\right\} .
\end{aligned}
$$

It is clear that $\omega_{X}$ is an IFP-equivalent intuitionistic fuzzy soft $h$-ideal over $S_{x y}$.

Notation 2. If $\Omega_{X}=\left\{\left(\left(\mu_{X}(x), \nu_{X}(x)\right) / x, \omega_{X}(x)\right): x \in\right.$ $\left.E, \omega_{X}(x) \in \operatorname{IF}(S)\right\}$ and $\Omega_{Y}=\left\{\left(\left(\mu_{Y}(y), \nu_{Y}(y)\right) / y, \omega_{Y}(y)\right): y \in\right.$ $\left.E, \omega_{Y}(y) \in \operatorname{IF}(S)\right\}$ are IFP-equivalent intuitionistic fuzzy soft $h$-ideals over hemiring $S$, then $\Omega_{X} \widetilde{\bigcap} \Omega_{Y}$ and $\Omega_{X} \widetilde{\cup} \Omega_{Y}$ are not always IFP-equivalent intuitionistic fuzzy soft $h$-ideals over $S$.

Example 25. Assume that $S=\mathbb{Z}_{3}, E=\left\{x_{1}, x_{2}, x_{3}\right\}$. Let $\Omega_{X}$ be an IFP-intuitionistic fuzzy soft $h$-ideal over $S$ defined by

$$
\begin{aligned}
X & =\left\{\frac{(0.3,0.5)}{x_{1}}, \frac{(0.3,0.5)}{x_{2}}, \frac{(0.2,0.6)}{x_{3}}\right\}, \\
\omega_{X}\left(x_{1}\right) & =\left\{\frac{(0.5,0.4)}{0}, \frac{(0.3,0.6)}{1},(0.3,0.6)\right\}, \\
\omega_{X}\left(x_{2}\right) & =\left\{\frac{(0.5,0.4)}{0}, \frac{(0.3,0.6)}{1},(0.3,0.6)\right\}, \\
\omega_{X}\left(x_{3}\right) & =\left\{\frac{(0.6,0.3)}{0}, \frac{(0.4,0.5)}{1},(0.4,0.5)\right\} .
\end{aligned}
$$

And let $\Omega_{Y}$ be an IFP-intuitionistic fuzzy soft $h$-ideal over $S$ defined by

$$
\begin{aligned}
Y & =\left\{\frac{(0.2,0.6)}{x_{1}}, \frac{(0.6,0.3)}{x_{2}}, \frac{(0.6,0.3)}{x_{3}}\right\}, \\
\omega_{Y}\left(x_{1}\right) & =\left\{\frac{(0.8,0.1)}{0}, \frac{(0.1,0.7)}{1},(0.1,0.7)\right\}, \\
\omega_{Y}\left(x_{2}\right) & =\left\{\frac{(0.6,0.3)}{0}, \frac{(0.2,0.5)}{1},(0.2,0.5)\right\}, \\
\omega_{Y}\left(x_{3}\right) & =\left\{\frac{(0.6,0.3)}{0}, \frac{(0.2,0.5)}{1},(0.2,0.5)\right\} .
\end{aligned}
$$

It is clear that $\omega_{X}$ and $\omega_{Y}$ are IFP-equivalent intuitionistic fuzzy soft $h$-ideals over $S$. We can see that

$$
\begin{aligned}
X \cap Y & =\left\{\frac{(0.2,0.6)}{x_{1}}, \frac{(0.3,0.5)}{x_{2}}, \frac{(0.2,0.6)}{x_{3}}\right\}, \\
\mu_{(X \cap Y)}\left(x_{1}\right) & =\mu_{(X \cap Y)}\left(x_{3}\right), \\
X \cup Y & =\left\{\frac{(0.3,0.5)}{x_{1}}, \frac{(0.6,0.3)}{x_{2}}, \frac{(0.6,0.3)}{x_{3}}\right\}, \\
\mu_{(X \cup Y)}\left(x_{2}\right) & =\mu_{(X \cup Y)}\left(x_{3}\right),
\end{aligned}
$$

but

$$
\begin{aligned}
& \omega_{(X \cap Y)}\left(x_{1}\right)=\left\{\frac{(0.5,0.4)}{0}, \frac{(0.1,0.7)}{1},(0.1,0.7)\right\}, \\
& \omega_{(X \cap Y)}\left(x_{3}\right)=\left\{\frac{(0.6,0.3)}{0}, \frac{(0.2,0.5)}{1},(0.2,0.5)\right\}, \\
& \omega_{(X \cup Y)}\left(x_{2}\right)=\left\{\frac{(0.6,0.3)}{0}, \frac{(0.3,0.5)}{1},(0.3,0.5)\right\}, \\
& \omega_{(X \cup Y)}\left(x_{3}\right)=\left\{\frac{(0.6,0.3)}{0}, \frac{(0.4,0.5)}{1},(0.4,0.5)\right\} .
\end{aligned}
$$

Then $\omega_{X} \widetilde{\cap} \omega_{Y}$ and $\omega_{X} \widetilde{U} \omega_{Y}$ are not IFP-equivalent intuitionistic fuzzy soft $h$-ideals over $S$.

Theorem 26. Let $\Omega_{X}=\left\{\left(\left(\mu_{X}(x), \nu_{X}(x)\right) / x, \omega_{X}(x)\right): x \in\right.$ $\left.A, \omega_{X}(x) \in I F(S)\right\}$ be an IFP-equivalent intuitionistic fuzzy soft $h$-ideal over hemiring $S$ and $\Omega_{Y}=\left\{\left(\left(\mu_{Y}(y), \nu_{Y}(y)\right) / y, \omega_{Y}(y)\right)\right.$ : $\left.y \in B, \omega_{Y}(y) \in I F(S)\right\}$ an IFP-intuitionistic fuzzy soft set over hemiring K. If $\Omega_{X}$ is IFP-intuitionistic fuzzy soft homomorphic to $\Omega_{Y}$, then $\Omega_{Y}$ is an IFP-equivalent intuitionistic fuzzy soft $h$ ideal over $K$.

Proof. Let $(f, g)$ be an IFP-intuitionistic fuzzy soft homomorphism from $\Omega_{X}$ to $\Omega_{Y}$. Since $\Omega_{X}$ is an IFP-equivalent intuitionistic fuzzy soft $h$-ideal over $S$, we have $\omega_{X}\left(x_{1}\right)=$ $\omega_{X}\left(x_{2}\right)$, if $\mu_{X}\left(x_{1}\right)=\mu_{X}\left(x_{2}\right)$ and $v_{X}\left(x_{1}\right)=v_{X}\left(x_{2}\right)$ for any $x_{1}, x_{2} \in A$. Now, for all $y_{1}, y_{2} \in B$, then there exist $x_{1}, x_{2} \in A$ with $g\left(x_{1}\right)=y_{1}, g\left(x_{2}\right)=y_{2}$. Since $\mu_{Y}\left(y_{1}\right)=$ $\mu_{Y}\left(g\left(x_{1}\right)\right)=\mu_{X}\left(x_{1}\right)$ and $\mu_{Y}\left(y_{2}\right)=\mu_{Y}\left(g\left(x_{2}\right)\right)=\mu_{X}\left(x_{2}\right)$, then $\mu_{Y}\left(y_{1}\right)=\mu_{Y}\left(y_{2}\right)$, and again, we have $\nu_{Y}\left(y_{1}\right)=v_{Y}\left(y_{2}\right)$. And then $\omega_{Y}\left(y_{1}\right)=\omega_{Y}\left(g\left(x_{1}\right)\right)=f\left(\omega_{X}\left(x_{1}\right)\right)=f\left(\omega_{X}\left(x_{2}\right)\right)=$ $\omega_{Y}\left(g\left(x_{2}\right)\right)=\omega_{Y}\left(y_{2}\right)$; hence $\Omega_{Y}$ must be an IFP-equivalent intuitionistic fuzzy soft $h$-ideal over $K$ as well.

Definition 27. Let $\Omega_{X}=\left\{\left(\left(\mu_{X}(x), \nu_{X}(x)\right) / x, \omega_{X}(x)\right): x \in\right.$ $\left.E, \omega_{X}(x) \in \operatorname{IF}(S)\right\}$ be an IFP-intuitionistic fuzzy soft $h$-ideal over hemiring $S$. Then $\Omega_{X}$ is said to be an IFP-increasing intuitionistic fuzzy soft $h$-ideal over $S$, if for any $x, y \in E$, $\mu_{X}(x) \leq \mu_{X}(y)$, and $\nu_{X}(x) \geq v_{X}(y)$, we have $\omega_{X}(x) \subseteq \omega_{X}(y)$, and $\Omega_{X}$ is said to be an IFP-decreasing intuitionistic fuzzy soft $h$-ideal over $S$, if for any $x, y \in E, \mu_{X}(x) \leq \mu_{X}(y)$, and $\nu_{X}(x) \leq \nu_{X}(y)$, we have $\omega_{X}(x) \supseteq \omega_{X}(y)$. 
Example 28. Let $S=\mathbb{Z}_{3}, E=\left\{x_{1}, x_{2}, x_{3}\right\}$, and $X$ be a fuzzy set over $E$ defined by

$$
\begin{aligned}
X & =\left\{\frac{(0.1,0.8)}{x_{1}}, \frac{(0.4,0.6)}{x_{2}}, \frac{(0.7,0.3)}{x_{3}}\right\}, \\
\omega_{X}\left(x_{1}\right) & =\left\{\frac{(0.2,0.6)}{0}, \frac{(0.1,0.8)}{1}, \frac{(0.1,0.8)}{2}\right\}, \\
\omega_{X}\left(x_{2}\right) & =\left\{\frac{(0.6,0.4)}{0}, \frac{(0.3,0.5)}{1}, \frac{(0.3,0.5)}{2}\right\}, \\
\omega_{X}\left(x_{3}\right) & =\left\{\frac{(0.9,0)}{0}, \frac{(0.7,0.2)}{1}, \frac{(0.7,0.2)}{2}\right\} .
\end{aligned}
$$

It is clear that $\omega_{X}$ is an IFP-increasing intuitionistic fuzzy soft hemiring over $S$.

Notation 3. If $\Omega_{X}=\left\{\left(\left(\mu_{X}(x), \nu_{X}(x)\right) / x, \omega_{X}(x)\right): x \in\right.$ $\left.E, \omega_{X}(x) \in \operatorname{IF}(S)\right\}$ and $\Omega_{Y}=\left\{\left(\left(\mu_{Y}(x), \nu_{Y}(x)\right) / x, \omega_{Y}(x)\right)\right.$ : $\left.x \in E, \omega_{Y}(x) \in \operatorname{IF}(S)\right\}$ are IFP-increasing intuitionistic fuzzy soft $h$-ideals over hemiring $S$, then $\Omega_{X} \widetilde{\bigcap} \Omega_{Y} \Omega_{X} \widetilde{\cup} \Omega_{Y}$ are not always an IFP-increasing intuitionistic fuzzy soft $h$ ideals over $S$.

Example 29. Assume that $S=\mathbb{Z}_{3}, E=\left\{x_{1}, x_{2}\right\}$. Let $X$ be an intuitionistic fuzzy set over $E$ defined by

$$
\begin{aligned}
X & =\left\{\frac{(0.3,0.6)}{x_{1}}, \frac{(0.5,0.2)}{x_{2}}\right\}, \\
\omega_{X}\left(x_{1}\right) & =\{(0.4,0.5),(0.1,0.6),(0.1,0.6)\}, \\
\omega_{X}\left(x_{2}\right) & =\{(0.6,0.2),(0.5,0.3),(0.5,0.3)\} .
\end{aligned}
$$

And let $Y$ be a fuzzy set over $E$ defined by

$$
\begin{aligned}
Y & =\left\{\frac{(0.9,0)}{x_{1}}, \frac{(0.4,0.3)}{x_{2}}\right\}, \\
\omega_{Y}\left(x_{1}\right) & =\{(0.6,0.2),(0.4,0.5),(0.4,0.5)\}, \\
\omega_{Y}\left(x_{2}\right) & =\{(0.3,0.5),(0.2,0.8),(0.2,0.8)\} .
\end{aligned}
$$

It is clear that $\omega_{X}$ and $\omega_{Y}$ are IFP-increasing intuitionistic fuzzy soft $h$-ideals over $S$. We can see that

$$
\begin{aligned}
& X \cap Y=\left\{\frac{(0.3,0.6)}{x_{1}}, \frac{(0.4,0.3)}{x_{2}}\right\}, \\
& X \cup Y=\left\{\frac{(0.9,0)}{x_{1}}, \frac{(0.5,0.2)}{x_{2}}\right\},
\end{aligned}
$$

but

$$
\begin{aligned}
& \omega_{(X \cap Y)}\left(x_{1}\right)=\{(0.4,0.5),(0.1,0.6),(0.1,0.6)\} ; \\
& \omega_{(X \cap Y)}\left(x_{2}\right)=\{(0.3,0.5),(0.2,0.8),(0.2,0.8)\} . \\
& \omega_{(X \cup Y)}\left(x_{1}\right)=\{(0.6,0.2),(0.4,0.5),(0.4,0.5)\} ; \\
& \omega_{(X \cup Y)}\left(x_{2}\right)=\{(0.6,0.2),(0.5,0.3),(0.5,0.3)\} .
\end{aligned}
$$

Then $\omega_{X} \widetilde{\cap} \omega_{Y}$ and $\omega_{X} \widetilde{\cup} \omega_{Y}$ are not IFP-increasing intuitionistic fuzzy soft $h$-ideal over $S$.
Theorem 30. Let $\Omega_{X}=\left\{\left(\left(\mu_{X}(x), \nu_{X}(x)\right) / x, \omega_{X}(x)\right): x \in\right.$ $\left.E, \omega_{X}(x) \in I F(S)\right\}$ be an IFP-increasing intuitionistic fuzzy soft $h$-ideal over hemiring $S$ and $\Omega_{Y}=\left\{\left(\left(\mu_{Y}(x), \nu_{Y}(x)\right) / x, \omega_{Y}(x)\right)\right.$ : $\left.x \in E, \omega_{Y}(x) \in I F(K)\right\}$ be an IFP-intuitionistic fuzzy soft set over hemiring $K$. If $\Omega_{X}$ is IFP-intuitionistic fuzzy soft homomorphic to $\Omega_{Y}$, then $\Omega_{Y}$ is an IFP-increasing intuitionistic fuzzy soft h-ideal over $S$.

Proof. Let $(f, g)$ be an IFP-intuitionistic fuzzy soft homomorphism from $\Omega_{X}$ to $\Omega_{Y}$. Since $\Omega_{X}$ is an IFP-increasing intuitionistic fuzzy soft $h$-ideal over $S$, for all $x_{1}, x_{2} \in A$, $\mu_{X}\left(x_{1}\right) \leq \mu_{X}\left(x_{2}\right)$, and $\nu_{X}\left(x_{1}\right) \geq \nu_{X}\left(x_{2}\right)$, we have $\omega_{X}\left(x_{1}\right) \subseteq$ $\omega_{X}\left(x_{2}\right)$. Now, for all $y_{1}, y_{2} \in B$, there exist $x_{1}, x_{2} \in A$ with $g\left(x_{1}\right)=y_{1}, g\left(x_{2}\right)=y_{2}$. Since $\mu_{Y}\left(y_{1}\right)=\mu_{Y}\left(g\left(x_{1}\right)\right)=\mu_{X}\left(x_{1}\right)$ and $\mu_{Y}\left(y_{2}\right)=\mu_{Y}\left(g\left(x_{2}\right)\right)=\mu_{X}\left(x_{2}\right)$, then $\mu_{Y}\left(y_{1}\right) \leq \mu_{Y}\left(y_{2}\right)$, and again, we have $\nu_{Y}\left(y_{1}\right) \geq \nu_{Y}\left(y_{2}\right)$. Hence, $\omega_{Y}\left(y_{1}\right)=$ $\omega_{Y}\left(g\left(x_{1}\right)\right)=f\left(\omega_{X}\left(x_{1}\right)\right) \subseteq f\left(\omega_{X}\left(x_{2}\right)\right)=\omega_{Y}\left(g\left(x_{2}\right)\right)=\omega_{Y}\left(y_{2}\right)$ and $\Omega_{Y}$ must be an IFP-increasing intuitionistic fuzzy soft $h$ ideal over $K$ as well.

\section{Decision Making Method on Intuitionistic Fuzzy $h$-Ideals}

The approximate function of an $\Omega$-set is an intuitionistic fuzzy set. The $\Omega_{\text {agg }}$ on the intuitionistic fuzzy sets is an operation by which several approximate functions of an $\Omega$ set are combined to produce a single intuitionistic fuzzy set that is the aggregate intuitionistic fuzzy set of the $\Omega$-set.

Definition 31. Let $\Omega_{X} \in \Omega(U)$ and $\operatorname{IF}(E)$ be a set of all the intuitionistic fuzzy sets over $E$. Then $\Omega$-aggregation operator, denoted by $\Omega_{\text {agg }}$, is defined by

$$
\begin{gathered}
\Omega_{\mathrm{agg}}: \operatorname{IF}(E) \times \Omega(U) \longrightarrow \operatorname{IF}(U), \\
\Omega_{\mathrm{agg}}\left(X, \Omega_{X}\right)=\Omega_{X}^{*},
\end{gathered}
$$

where

$$
\Omega_{X}^{*}=\left\{\frac{\left(\mu_{\Omega_{X}^{*}}(u), v_{\Omega_{X}^{*}}(u)\right)}{u} \mid u \in U\right\},
$$

which is an intuitionistic fuzzy set over $U$. The value $\Omega_{X}^{*}$ is called aggregate intuitionistic fuzzy set of $\Omega_{X}$. Here the membership degree $\mu_{\Omega_{X}^{*}}(u)$ and nonmembership degree $v_{\Omega_{X}^{*}}(u)$ of $u$ are defined as follows:

$$
\begin{aligned}
& \mu_{\Omega_{X}^{*}}(u)=\frac{1}{|E|} \sum_{x \in E} \mu_{X}(x) \mu_{\omega_{X}(x)}(u), \\
& v_{\Omega_{X}^{*}}(u)=\frac{1}{|E|} \sum_{x \in E} v_{X}(x) v_{\omega_{X}(x)}(u),
\end{aligned}
$$

where $|E|$ is the cardinality of $E$.

Theorem 32. Let $\Omega_{X}=\left\{\left(\left(\mu_{X}(x), \nu_{X}(x)\right) / x, \omega_{X}(x)\right): x \in\right.$ $\left.E, \omega_{X}(x) \in I F(S)\right\}$ be an IFP-intuitionistic fuzzy soft $h$-ideal over $S$. Then the aggregate intuitionistic fuzzy set $\Omega_{X}^{*}$ of $\Omega_{X}$ is an intuitionistic fuzzy $h$-ideal of $S$. 
Proof. For any $x \in E, \omega_{X}(x)$ is an intuitionistic fuzzy $h$-ideal of $S$. That is, for all $s_{1}, s_{2} \in S$, $\mu_{\omega_{X}(x)}\left(s_{1}+s_{2}\right) \geq \min \left\{\mu_{\omega_{X}(x)}\left(s_{1}\right), \mu_{\omega_{X}(x)}\left(s_{2}\right)\right\}, \mu_{\omega_{X}(x)}\left(s_{1} s_{2}\right) \geq$ $\max \left\{\mu_{\omega_{X}(x)}\left(s_{1}\right), \mu_{\omega_{X}(x)}\left(s_{2}\right)\right\}$ and for all any $a, b, s, z \in S$, $s+a+z=b+z$ implies $\mu_{\omega_{X}(x)}(s) \geq \min \left\{\mu_{\omega_{X}(x)}(a), \mu_{\omega_{X}(x)}(b)\right\}$. Then

$$
\begin{aligned}
& \mu_{\Omega_{X}^{*}}\left(s_{1}+s_{2}\right) \\
& =\frac{1}{|E|} \sum_{x \in E} \mu_{X}(x) \mu_{\omega_{X}(x)}\left(s_{1}+s_{2}\right) \\
& \geq \min \left\{\frac{1}{|E|} \sum_{x \in E} \mu_{X}(x) \mu_{\omega_{X}(x)}\left(s_{1}\right), \frac{1}{|E|} \sum_{x \in E} \mu_{X}(x) \mu_{\omega_{X}(x)}\left(s_{2}\right)\right\} \\
& =\min \left\{\mu_{\Omega_{X}^{*}}\left(s_{1}\right), \mu_{\Omega_{X}^{*}}\left(s_{2}\right)\right\} .
\end{aligned}
$$

In the same way, we can obtain: $\mu_{\Omega_{X}^{*}}\left(s_{1} s_{2}\right) \geq$ $\max \left\{\mu_{\Omega_{X}^{*}}\left(s_{1}\right), \mu_{\Omega_{X}^{*}}\left(s_{2}\right)\right\}, \mu_{\Omega_{X}^{*}}(s) \geq \min \left\{\mu_{\Omega_{X}^{*}}(a), \mu_{\Omega_{X}^{*}}(b)\right\}$ and $v_{\Omega_{X}^{*}}\left(s_{1}+s_{2}\right) \leq \max \left\{v_{\Omega_{X}^{*}}\left(s_{1}\right), v_{\Omega_{X}^{*}}\left(s_{2}\right)\right\}, v_{\Omega_{X}^{*}}\left(s_{1} s_{2}\right) \leq$ $\min \left\{v_{\Omega_{X}^{*}}\left(s_{1}\right), v_{\Omega_{X}^{*}}\left(s_{2}\right)\right\}$, and $v_{\Omega_{X}^{*}}(s) \leq \max \left\{v_{\Omega_{X}^{*}}(a), v_{\Omega_{X}^{*}}(b)\right\}$, which implies $\Omega_{X}^{*}$ is an intuitionistic fuzzy $h$-ideal of $S$.

Notation 4. The above $\Omega_{X}^{*}$ is called an aggregate intuitionistic fuzzy (left/right) $h$-ideal of IFP-intuitionistic fuzzy soft (left/right) $h$-ideal $\Omega_{X}$.

Example 33. Let $S$ be a full matrix hemiring, written by $M_{n}, A$ a lower triangular matrix, and $B$ a lower diagonal matrix. And let $E=\{a, b\}$, the parameters $a, b$ stand for "lower triangular" and "diagonal," respectively. If $X$ is an intuitionistic fuzzy set over $E$ defined by

$$
\mu_{X}(x)=\left\{\begin{array}{ll}
0.6, & x=a, \\
0.2, & x=b,
\end{array} \quad v_{X}(x)=1-\mu_{X}(x) .\right.
$$

Define $\omega_{X}$ by

$\mu_{\omega_{X}(a)}(r)= \begin{cases}0, & r \text { is not alower triangular matrix; } \\ 1, & r \text { is alower triangular matrix. }\end{cases}$

$\mu_{\omega_{X}(b)}(r)= \begin{cases}0, & r \text { is not adiagonal matrix } \\ 1, & r \text { is adiagonal matrix. }\end{cases}$

And $\nu_{\omega_{X}}(r)=1-\mu_{\omega_{X}}(r)$.

We can verify that $\Omega_{X}^{*}$ is an intuitionistic fuzzy left $h$-ideal of $M_{n}$. Then $\Omega_{X}^{*}$ is called an aggregate intuitionistic fuzzy left $h$-ideal of the $\Omega_{X}$.

For the above Example, we consider an intuitionistic fuzzy $h$-ideal of $\Omega_{X}^{*}$. Certainly, we can take into consideration the general condition on $\Omega_{X}^{*}$.

Definition 34. In this definition, one can construct a decisionmaking method by the following algorithm.

(1) Construct an $\Omega$-set over $U$,

(2) Find the aggregate intuitionistic fuzzy set $\Omega_{X}^{*}$ of $\Omega_{X}$,
(3) Find $\max (u)=\max \left\{\mu_{\Omega_{X}^{*}}(u) \mid u \in U\right\}$ and $\min (v)=$ $\min \left\{v_{\Omega_{X}^{*}}(v) \mid v \in U\right\}$,

(4) Find $\alpha \in[0,1]$ such that $(\max (u), \alpha) / u \in \Omega_{X}^{*}$ and $\beta \in[0,1]$ such that $(\beta, \min (v)) / v \in \Omega_{X}^{*}$,

(5) Find $\max (u) /(\max (u)+\alpha)=\alpha^{\prime}$ and $\beta /(\min (u)+\beta)=$ $\beta^{\prime}$

(6) Opportune element of $U$ is denoted by $\operatorname{Opp}(u)$ and it is chosen as follows:

$$
\operatorname{Opp}(u)= \begin{cases}u, & \text { if } \alpha^{\prime}>\beta^{\prime} \\ v, & \text { if } \alpha^{\prime}<\beta^{\prime}\end{cases}
$$

Example 35. In this example, we present an application for the $\Omega$-decision-making method.

Let us assume that people want to buy a lottery tickets. Here, we consider only five figures, and they are defined as $S=\mathbb{Z}_{5}=\{0,1,2,3,4\}$. The buyer considers a set of parameters, $E=\left\{x_{1}, x_{2}, x_{3}\right\}$. For $i=1,2,3$, the parameters $x_{i}$ stand for "preference," "frequency of occurrence," and "mutual interference," respectively.

After a serious discussion, each figure from point of view of the foals and the constraint according to a chosen subset $X=\left\{(0.6,0.3) / x_{1},(0.8,0.2) / x_{2}\right.$, and $\left.(0.7,0.2) / x_{3}\right\}$ of $E$. Finally, the buyer constructs the following $\Omega$-set over $S$.

(1) Let the constructed $\Omega$-set, $\Omega_{X}$, be as follows:

$$
\begin{aligned}
\Omega_{X} & \left\{\left(\frac{(0.6,0.3)}{x_{1}},\right.\right. \\
& \left.\left\{\frac{(0.2,0.6)}{0}, \frac{(0.7,0.3)}{1}, \frac{(0.4,0.5)}{2}, \frac{(0.8,0.1)}{3}, \frac{(0.1,0.6)}{4}\right\}\right), \\
& \left(\frac{(0.8,0.2)}{x_{2}},\right. \\
& \left.\left\{\frac{(0.4,0.6)}{0}, \frac{(0.9,0.1)}{1}, \frac{(0.5,0.4)}{2}, \frac{(0.6,0.2)}{3}, \frac{(0.6,0.3)}{4}\right\}\right), \\
& \left(\frac{(0.7,0.2)}{x_{3}},\right. \\
& \left.\left.\left\{\frac{(0.2,0.6)}{0}, \frac{(0.6,0.3)}{1}, \frac{(0.7,0.1)}{2}, \frac{(0.7,0.2)}{3}, \frac{(0.6,0.3)}{4}\right\}\right)\right\} .
\end{aligned}
$$

(2) The aggregate intuitionistic fuzzy set can be found as

$$
\begin{aligned}
\Omega_{X}^{*}=\left\{\frac{(0.193,0.14)}{0}, \frac{(0.52,0.056)}{1}, \frac{(0.376,0.083)}{2},\right. \\
\left.\frac{(0.483,0.036)}{3}, \frac{(0.32,0.1)}{4}\right\} .
\end{aligned}
$$


(3) $\max (u)=0.52$ and $\min (v)=0.036$.

(4) $(0.52,0.056) / 1 \in \Omega_{X}^{*}$ and $(0.483,0.036) / 3 \in \Omega_{X}^{*}$.

(5) $\alpha^{\prime}=0.52 /(0.52+0.056)=0.903$ and $\beta^{\prime}=$ $0.483 /(0.483+0.036)=0.931$.

(6) Since $\alpha^{\prime}<\beta^{\prime}, \operatorname{Opp}(u)=3$.

In the above Example, the decision-making method only gives one-digit number from 0 to 4 . By the similar way, we can arrange many digits from 0 to 9 .

\section{Conclusion}

In the present paper, we showed the basic results of IFPintuitionistic fuzzy soft $h$-ideal. And then we defined IFPintuitionistic fuzzy soft homomorphisms (isomorphisms), IFP-equivalent intuitionistic fuzzy soft $h$-ideals, and IFPincreasing intuitionistic fuzzy soft $h$-ideals and discussed some properties of them. Finally, we investigate aggregate intuitionistic fuzzy $h$-ideals of hemirings based on decision making.

As an extension of this work, one could apply the method to solve the related problems in applied mathematics and information sciences.

\section{Conflict of Interests}

The authors declare that there is no conflict of interests regarding the publication of this paper.

\section{Acknowledgment}

The authors are very grateful to referees for their valuable comments and suggestions for improving this paper. This research is partially supported by a Grant of National Natural Science Foundation of China (61175055), Natural Science Foundation of Hubei Province (2012FFB01101), and Natural Science Foundation of Education Committee of Hubei Province (D20131903).

\section{References}

[1] D. R. LaTorre, "On $h$-ideals and $k$-ideals in hemirings," Publicationes Mathematicae Debrecen, vol. 12, pp. 219-226, 1965.

[2] Y. B. Jun, M. A. Öztürk, and S. Z. Song, "On fuzzy $h$-ideals in hemirings," Information Sciences, vol. 162, no. 3-4, pp. 211-226, 2004.

[3] J. Zhan and W. A. Dudek, "Fuzzy $h$-ideals of hemirings," Information Sciences. An International Journal, vol. 177, no. 3, pp. 876-886, 2007.

[4] Y.-Q. Yin, X.-K. Huang, D.-H. Xu, and F. Li, "The characterization of $h$-semisimple hemirings," International Journal of Fuzzy Systems, vol. 11, no. 2, pp. 116-122, 2009.

[5] Y. Yin and H. Li, "The characterizations of $h$-hemiregular hemirings and $h$-intra-hemiregular hemirings," Information Sciences, vol. 178, no. 17, pp. 3451-3464, 2008.

[6] X. Ma and J. Zhan, "On fuzzy $h$-ideals of hemirings," Journal of Systems Science \& Complexity, vol. 20, no. 3, pp. 470-478, 2007.
[7] X. Ma and J. Zhan, "Generalized fuzzy $h$-bi-ideals and $h$-quasiideals of hemirings," Information Sciences, vol. 179, no. 9, pp. 1249-1268, 2009.

[8] X. Ma, Y. Yin, and J. Zhan, "Characterizations of $h$-intra- and $h$ quasi-hemiregular hemirings," Computers \& Mathematics with Applications, vol. 63, no. 4, pp. 783-793, 2012.

[9] J. Zhan and K. P. Shum, "Intuitionistic $(S, T)$-fuzzy $h$-ideals of hemirings," East Asian Mathematics, vol. 22, pp. 93-109, 2006.

[10] W. A. Dudek, M. Shabir, and R. Anjum, "Characterizations of hemirings by their $h$-ideals," Computers \& Mathematics with Applications, vol. 59, no. 9, pp. 3167-3179, 2010.

[11] D. Molodtsov, "Soft set theory-first results," Computers \& Mathematics with Applications, vol. 37, no. 4-5, pp. 19-31, 1999.

[12] M. I. Ali, "Another view on reduction of parameters in soft sets," Applied Soft Computing Journal, vol. 12, no. 6, pp. 1814-1821, 2012.

[13] M. I. Ali, F. Feng, X. Liu, W. K. Min, and M. Shabir, "On some new operations in soft set theory," Computers \& Mathematics with Applications, vol. 57, no. 9, pp. 1547-1553, 2009.

[14] M. I. Ali, M. Shabir, and M. Naz, "Algebraic structures of soft sets associated with new operations," Computers \& Mathematics with Applications, vol. 61, no. 9, pp. 2647-2654, 2011.

[15] F. Feng, Y. B. Jun, X. Liu, and L. Li, "An adjustable approach to fuzzy soft set based decision making," Journal of Computational and Applied Mathematics, vol. 234, no. 1, pp. 10-20, 2010.

[16] F. Feng, Y. B. Jun, and X. Zhao, "Soft semirings," Computers \& Mathematics with Applications, vol. 56, no. 10, pp. 2621-2628, 2008.

[17] X. Liu, D. Xiang, and J. Zhan, "Fuzzy isomorphism theorems of soft rings," Neural Computing and Applications, vol. 21, no. 2, pp. 391-397, 2012.

[18] X. Ma and J. Zhan, "Characterizations of three kinds of hemirings by fuzzy soft $h$-ideals," Journal of Intelligent \& Fuzzy Systems, vol. 24, no. 3, pp. 535-548, 2013.

[19] A. R. Roy and P. K. Maji, "A fuzzy soft set theoretic approach to decision making problems," Journal of Computational and Applied Mathematics, vol. 203, no. 2, pp. 412-418, 2007.

[20] N. Çaĝman, F. Citak, and S. Enginogĝlu, "Fuzzy parameterized fuzzy soft set theory and its applications," Turkish Journal of Fuzzy Systems, vol. 1, pp. 21-35, 2010.

[21] F. Karaaslan, N. Çaĝman, and S. Yilmaz, "IFP-intuitionistic fuzzy soft set theory and its applications," In press.

[22] L. A. Zadeh, "Fuzzy sets," Information and Computation, vol. 8, pp. 338-353, 1965.

[23] K. T. Atanassov, "Intuitionistic fuzzy sets," Fuzzy Sets and Systems, vol. 20, no. 1, pp. 87-96, 1986. 


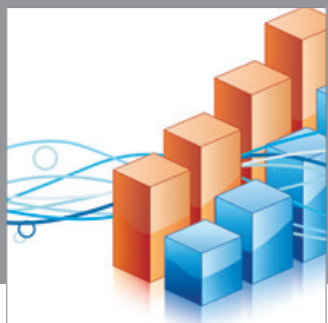

Advances in

Operations Research

mansans

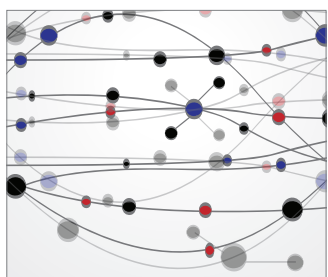

The Scientific World Journal
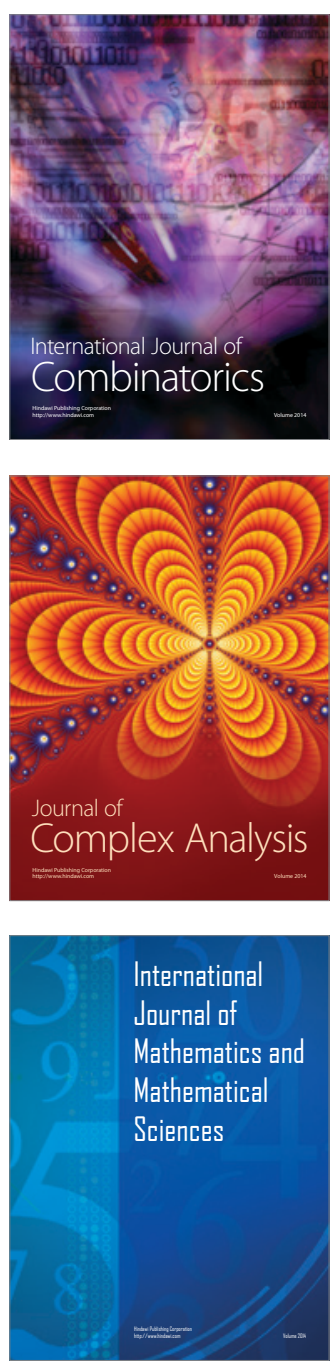
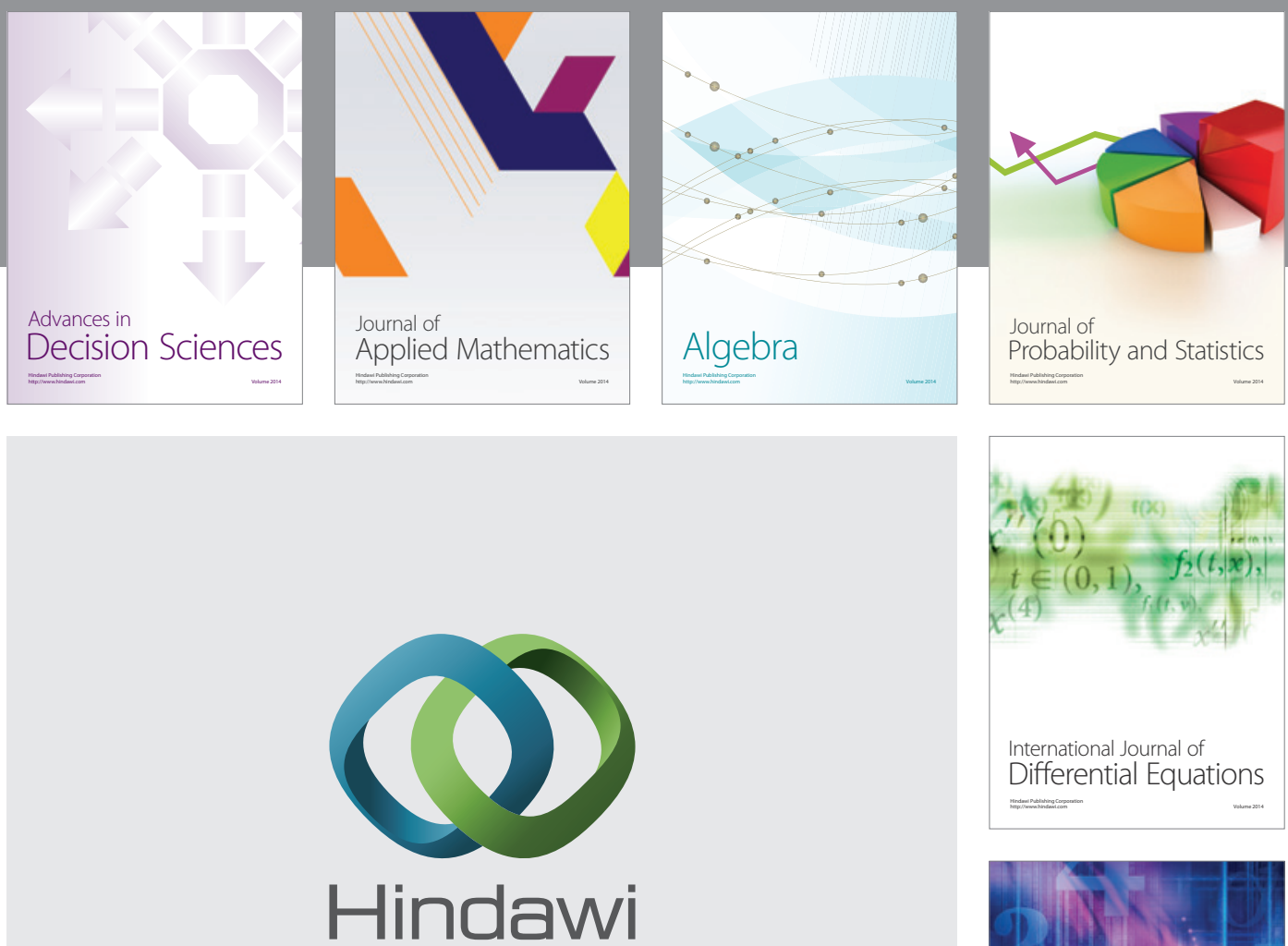

Submit your manuscripts at http://www.hindawi.com
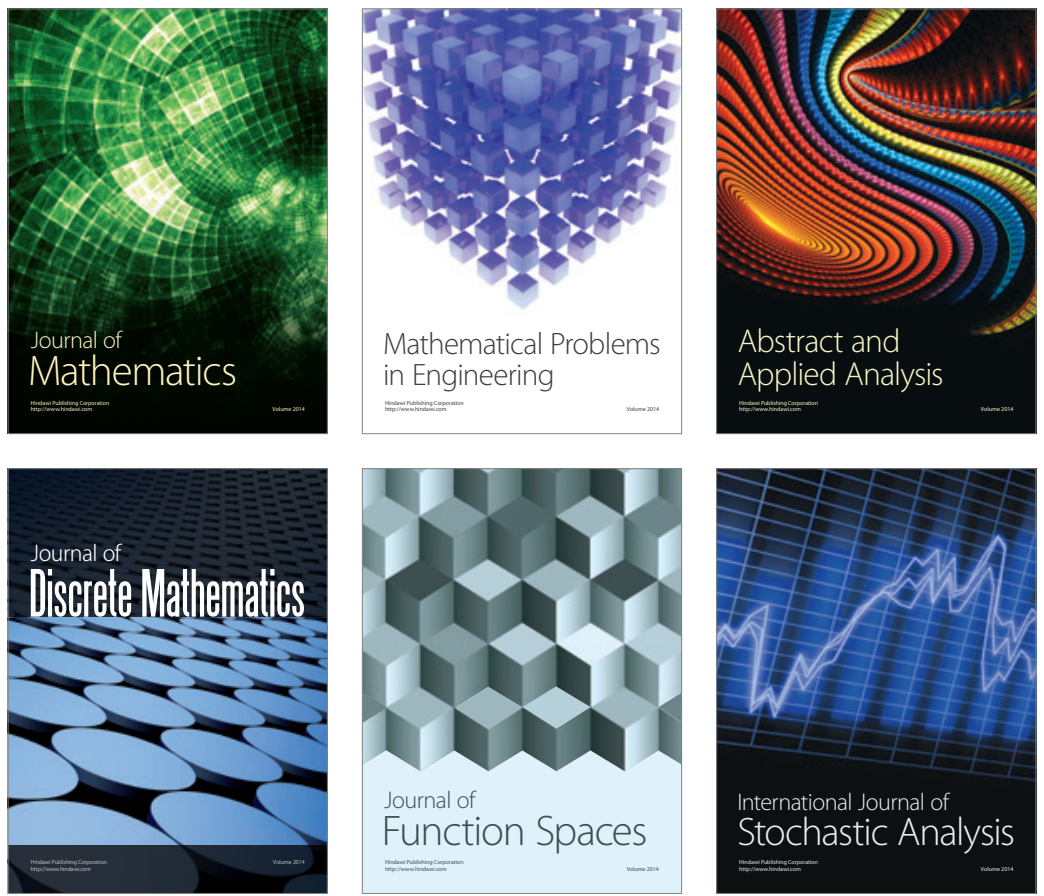

Journal of

Function Spaces

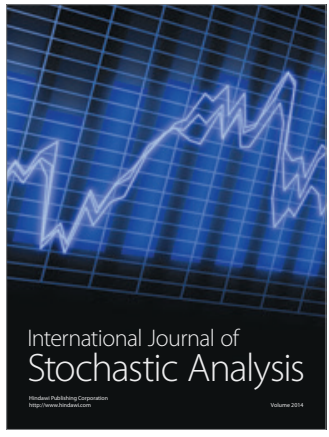

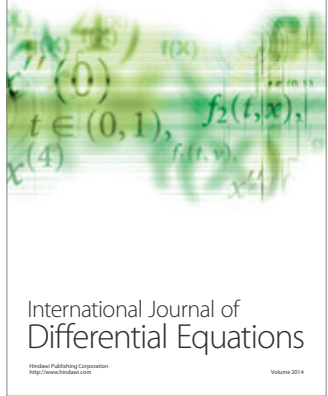
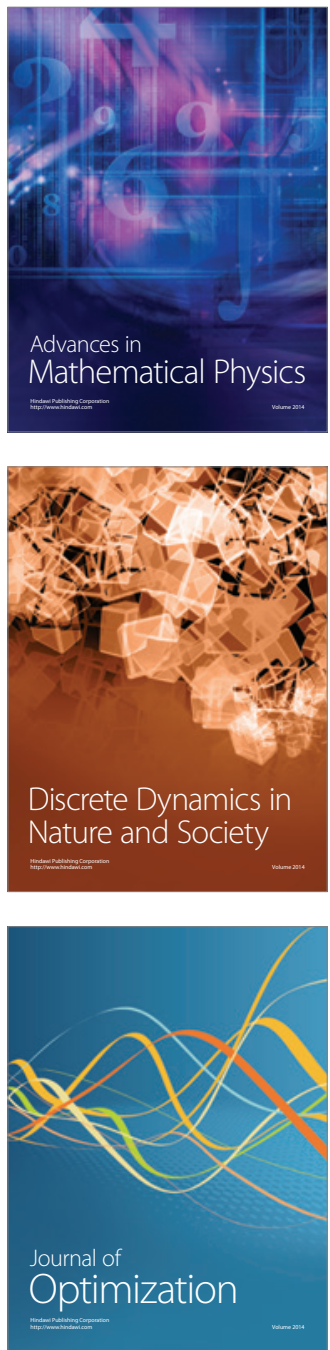